\title{
A New Method for Quantitative Sampling of the Sea-bottom.
}

\author{
By \\ O. D. Hunt, B.Sc., \\ Assistant Naturalist at the Plymouth Laboratory.
}

With 2 Figures in the Text.

The apparatus described in this paper has been specially designed for the taking of samples in cases where it is important that the finer constituents of the sample should be adequately retained. In commencing a study of the micro-fauna and micro-flora of the sea-bottom near Plymouth the writer found that, in samples taken by the "Petersen" (2) $1 / 10$ sq. metre bottom-sampler, or by the conical dredge, the silt and finest material was liable to ke washed away to a variable and nonmeasurable extent while hauling the apparatus. Quantitative observations on very small organisms were judged impossible by these methods and attention was directed towards devising an apparatus which would retain the finer portions of the deposits.

Sounding-tubes of the "Naumann " and "Lundqvist" types, described by Lundqvist (1), though very efficient in sampling soft bottoms of clay or mud, cannot be used for sampling sands and gravels, which, besides being too resistant to the boring of the tube, are not sufficiently cohesive to be retained as a sample by this method.

A method has now been devised which, in brief essentials, is as follows. A metal chamber, hermetically sealed by a glass diaphragm, is lowered to the bottom, on reaching which the diaphragm is automatically broken. The pressure of the overlying water-column forces into the chamber a sample of the bottom, which is prevented by a "trap" device from escaping when the apparatus is raised. For the original suggestion of utilising the pressure of the overlying water-column for the separation of a sample from the bottom, as well as for much help and advice in the experimental design of the apparatus, the writer is indebted to Dr. G. P. Bidder.

A photograph of the apparatus, which may be referred to as a "Vacuum Grab," is shown in Text Fig. 2. It is best described in detail by reference 
to the drawing (Text Fig. 1). It is made throughout of brass. The cylindrical "pressure-chamber," $\mathrm{PC}$, is provided above with a lug, L, by which it can be shackled to a sounding-wire, and is closed below by a massive screw-stopper, $\mathrm{S}$, the joint being made hermetically tight by a rubber washer, W. This stopper, which carries the remainder of the apparatus, is bored through its centre, the upper half of the boring being of less diameter than the lower. A shoulder is so formed with a downwardly-directed, horizontal face, SF. This face is accurately turned and smoothly finished. To the upper bore is permanently fitted the "trap-tube," TT, which projects into the "pressure-chamber" and is turned through a right-angle at its upper end. The lower, larger bore is threaded and into it is screwed the sleeve, Sl, which carries the sliding "sampling-tube," ST. A series of ports, P, is cut in the sides of the sleeve, towards its upper end, in order to provide escapement for water entering the mouth of the "sampling-tube" during descent. The "sampling-tube" slides easily within the sleeve, the extent of its movment being limited by two screws, Sc, which are fitted, one on each side into the "sampling-tube" and slide in vertical slots, VS, in the sleeve. When the "sampling-tube" is in position at the upper limit of its sliding range, its sides completely close the ports in the sleeve. The lower end of the "sampling-tube" is expanded into a bell-shaped mouth, while at its upper end is the bayonet-striker, BS, a narrow, bayonet-shaped piece, projecting inwards and upwards to a striking-point which lies in the centre of the bore. It will be seen that the only entrance to the "pressure-chamber" is by way of the "sampling-tube" and "traptube." Between these two the glass diaphragm, GD, is inserted, being sealed by a stiff vaseline preparation to the shoulder-face, SF.

In practice, the sleeve, with "sampling-tube," is detached and the glass disc inserted. The sleeve is then screwed in, hand-tight, and the instrument sent to the bottom at the end of a sounding-wire. When the lower end of the "sampling-tube "reaches the bottom the "samplingtube " is arrested, but, owing to the sliding sleeve, the remainder of the apparatus continues to sink until the glass disc touches and is broken by the bayonet-striker. At the moment of breakage the pressure inside the chamber is equal to atmospheric pressure, whilst that outside is equal to atmospheric pressure plus the pressure of the overlying watercolumn, which is approximately equivalent to an additional atmosphere's pressure for every 10 metres of depth. On breakage of the glass, therefore, a pressure equal to that of the water-column overlying the sea-bottom operates to force a sample from the area marked out by the lower edge of the " sampling-tube " up and through the trap-tube into the "pressure-chamber," where it falls to the bottom and cannot escape. 


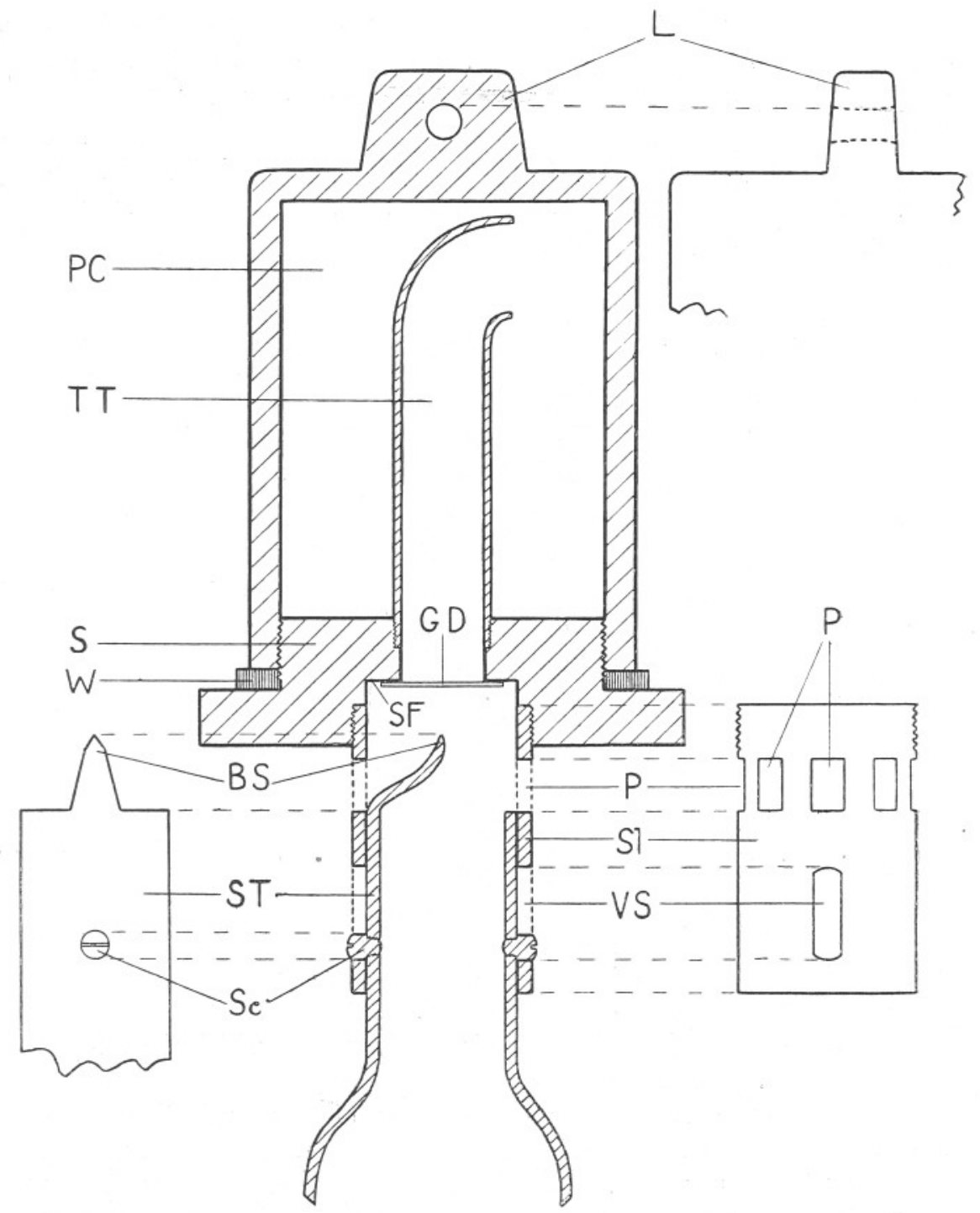

Fıc. 1.-Section of "Vacuum Grab," side view, with projections illustrating lug, sleeve, and "sampling-tube." $\times \frac{2}{5}$

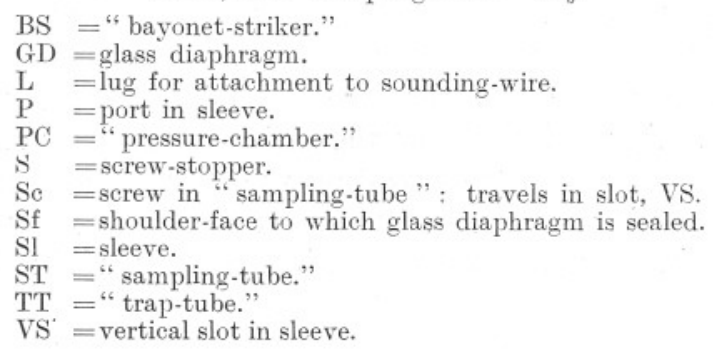


Samples taken in this way, whose volume is too small to rise above the level of the upper opening of the "trap-tube," are subject to no loss of their finer constituent particles. With larger samples, however, there is a slight loss due to removal of fine particles in suspension in such part of the water-content as lies above this-tevel. This loss, however, can be corrected for, because it varies in constant proportion to the

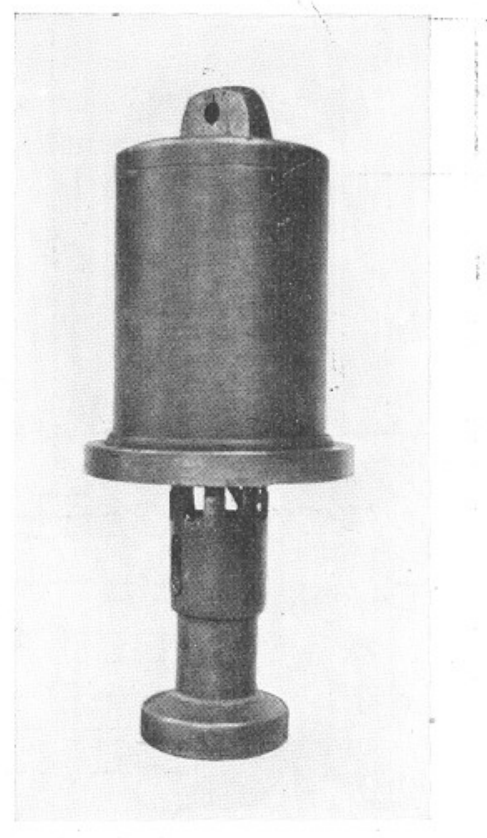

FIc. 2.-Photograph of "Vacuum Grab."

volume of the sample, and, therefore, to the depth at which the sample has been taken.

The samples taken by the above method enable a quantitative gravimetric and volumetric analysis of the constituents. To obtain values, however, relating to the area of bottom sampled it is necessary to know the relation between surface area and volume in samples of respectively different constitution and taken at dififerent depths. To this end a series of experiments was conducted in the laboratory and at sea, in which samples were taken of artificial bottoms of known constitution, and in which the size of the sample was varied by varying either the pressure or the depth. In the laboratory the different pressures necessary for sampling were obtained by exhausting the "pressure-chamber" to a varying extent. In the experiments at sea the depth was varied. The materials used in constructing the artificial bottoms varied from fine sand 
to shell-gravel in which the largest particles did not, on the average, exceed $1 \mathrm{~cm}$. in diameter. The bottoms were constructed by arranging successive layers of material, each $1 \mathrm{~cm}$. deep, in a straight-sided bucket. In each layer small, coloured beads of different shape and colours were so arranged that when a sample had been taken it was possible, by counting the numbers of each kind of bead in the sample, to reconstruct the area of each layer sampled. It was also possible to estimate the volume by this method, and the degree of correspondence between this estimated volume and the measured volume afforded a useful test of the accuracy of the method. The result of these experiments has been to show that, as expected on theoretical grounds, the area sampled does not vary materially either with different types of soil or with different pressures. The shape of the sample is approximately a cylinder of the same area of cross-section as that of the lower end of the "sampling-tube," the lower portion of the cylinder being rounded off or cup-shaped. With increasing pressure the instrument samples from a greater depth in the bottom-material, but not from a wider area : it digs, or rather sucks, a deeper though not a wider hole. These experiments have also shown that, as was suspected, there is a wafting away of a certain amount of surface material owing to disturbance of the immediately overlying water by the descending "sampling-tube." The ports cut in the sides of the sleeve, which are open during descent, but are closed automatically by the sliding mechanism on reaching the bottom, minimise this disturbance and limit the wafting away to a peripheral area. The experiments have demonstrated the extent of the area affected, so that the surface area truly sampled is known with sufficient accuracy. The details of these experiments, which are not yet completed, will be given in a later paper dealing with the samples obtained.

The above-described model of a "Vacuum Grab" has been specially designed for working under the submarine conditions which exist in the neighbourhood of Plymouth. It has been worked satisfactorily at all depths tried, which range from 10 metres to 70 metres. For working in depths up to 50 metres, a thickness of 1.5 millimetres for the glass diaphragm was found sufficient: from 50 to 70 metres, a thickness of 2.0 millimetres proved adequate. The "Vacuum Grab" is particularly suitable for work on bottoms of sand and fine gravel, samples of which as previously pointed out, cannot be obtained with the ordinary soundingtube. For sampling muds and oozes, however, whose resistance to the sounding-tube is small, and whose cohesive properties are great, the sounding-tube would be more satisfactory than the present apparatus, for it possesses the great advantage of taking a stratified sample. For this reason the "Vacuum Grab" is not likely to compete with the sounding-tube as a bottom-sampler in the deep sea, where oozes and 
0. D. HUNT.

clays constitute the bottom; but in shallow and moderately shallow waters, where it is required to work over a greater range of types of bottom, it is, so far as the writer is aware, the only means yet described of obtaining quantitative samples with respect to the finer constituents.

\section{AUTHORS CITED.}

1. Lundqvist, G. 1925. Methoden zur Untersuchung der Entwicklungsgeschichte der Seen. Abderhalden, Handbuch der bio. logischen Arbeitsmethoden, Abt. IX, Teil 2, p. 427.

2. Petersen, C. G. J. and Boysen Jensen. 1911. Valuation of the Sea, I. Report XX from the Danish Biological Station. 